\title{
Physiological analysis and transcriptome comparison of two muskmelon (Cucumis melo L.) cultivars in response to salt stress
}

\author{
L.M. Wang ${ }^{1 *}$, L.D. Zhang ${ }^{1 *}$, J.B. Chen ${ }^{1}$, D.F. Huang ${ }^{1}$ and Y.D. Zhang ${ }^{1,2}$ \\ ${ }^{1}$ School of Agriculture and Biology, Shanghai Jiao Tong University, Shanghai, \\ China \\ ${ }^{2}$ Key Laboratory of Urban Agriculture (South), Ministry of Agriculture, \\ Shanghai, China \\ *These authors contributed equally to this study. \\ Corresponding author: Y.D. Zhang \\ E-mail: zhyd@sjtu.edu.cn \\ Genet. Mol. Res. 15 (3): gmr.15038738 \\ Received April 27, 2016 \\ Accepted June 29, 2016 \\ Published September 19, 2016 \\ DOI http://dx.doi.org/10.4238/gmr.15038738
}

Copyright (C) 2016 The Authors. This is an open-access article distributed under the terms of the Creative Commons Attribution ShareAlike (CC BY-SA) 4.0 License.

\begin{abstract}
Melon (Cucumis melo L.) is an important vegetable crop that ranks second in salt tolerance among the Cucurbitaceae. Previous studies on the two muskmelon cultivars 'Bing XueCui' (BXC) and 'Yu Lu' (YL) revealed that they had different characteristics under salt stress, but the molecular basis underlying their different physiological responses is unclear. Here, we combined a physiological study with a genome-wide transcriptome analysis to understand the molecular basis of genetic variation that responds to salt stress in the melon. BXC performed better under salt stress than YL in terms of biomass and photosynthetic characteristics, because it exhibited less reduction in transpiration rate, net photosynthesis rate, and stomatal conductance under 150-mM NaCl stress than YL. A transcriptome comparison of the
\end{abstract}


leaves of the cultivars revealed that 1171 genes responded to salt stress in BXC while 1487 genes were identified as salt-stress-responsive in YL. A real-time polymerase chain reaction analysis of 12 of the responsive genes revealed that there was a strong, positive correlation with RNA sequencing data. The genes were involved in several pathways, including photosynthesis, the biosynthesis of secondary metabolites, metabolism, and plant hormone signal transduction, and their expression levels differed between the two cultivars in response to salt stress. This study provides a molecular perspective of two melon cultivars in response to salt stress, and its results could be used to investigate the complex molecular mechanisms underlying salt tolerance in the melon.

Key words: Melon (Cucumis melo L.); RNA sequencing; Salt stress; Physiological analysis; Transcriptome comparison

\section{INTRODUCTION}

High salinity is a major abiotic stressor that affects agricultural industries. Mild salt stress primarily affects plant development, agronomy traits, and agricultural productivity, and extremely high salinity causes plant death (Godfray et al., 2010). In addition, climate change and declining water quality are of great concern, and have resulted in land degradation due to high salt levels in the soil.

Melon (Cucumis melo L.) is an important global vegetable crop. Although salinity generally depresses melon vegetative growth and fruit development and quality, some melon cultivars can survive in relatively high saline soils. Indeed, the fruit quality of some melon cultivars can be improved by irrigation with mildly saline water during fruit set, and the salt tolerance capability of melon ranks second in the Cucurbitaceae (Sivritepe et al., 2005); therefore, it may be useful in the phytoremediation of saline land. Previous studies have investigated the physiological features of melon under salt treatments, and correlations between some physiological indices and salinity have been established (del Amor et al., 2000). Recent studies have investigated the molecular mechanisms of muskmelon salt tolerance in a saline environment (Zhang et al., 2011; Wei et al., 2013), which could be used to exploit salinized soil.

Whether plants in general can survive under saline conditions depends, to a large extent, on their ability to exclude salt from their shoots, or to tolerate high concentrations of salt in their leaves (tissue tolerance), which involves the ability to maintain ionic homeostasis, particularly of $\mathrm{K}^{+}, \mathrm{Na}^{+}$, and $\mathrm{Cl}^{-}$(Sun et al., 2009). Maintaining a high $\mathrm{K}^{+} / \mathrm{Na}^{+}$ratio in the cytosol of plant cells has been proven to be a key feature of salt-tolerant plants, and is often suggested as a potential screening tool for plant breeders (Tester and Davenport, 2003). The correlation between ion fluxes (e.g., $\mathrm{Na}^{+}, \mathrm{K}^{+}$, or $\mathrm{Cl}^{-}$) in the roots or leaf mesophyll and the capacity for saline resistance has been investigated using non-invasive micro-test technology (NMT), which is a relatively novel, reliable, and rapid physiological marker for selecting salttolerant plants (Cuin et al., 2008). Under saline conditions, optimizing water-use efficiency and protecting photosynthetic machinery are the two fundamental mechanisms for tolerating high-salt conditions (Munns and Tester, 2008). Stomata play a key role in gas exchange for photosynthesis, and in minimizing transpiration (Sirichandra et al., 2009). The bulk of the osmotic content that drives stomatal movements depends upon the intracellular concentrations

Genetics and Molecular Research 15 (3): gmr.15038738 
of inorganic and organic ions $\left(\mathrm{K}^{+}, \mathrm{Na}^{+}, \mathrm{Cl}^{-}\right.$, and malate $\left.{ }^{2-}\right)$ and sugar, and involves several ion channels and transporters. Previously, we cloned a K-channel gene, melon inward rectifying $K^{+}$channel (MIRK), from a mildly salt-tolerant melon cultivar. MIRK is mainly expressed in guard cells (Zhang et al., 2011), and may play a role in modulating stomatal aperture and contributes to high salt tolerance (Wang et al., 2013). However, the relationship between ion fluxes, or specific ion channel(s)/transporter(s) of guard cells, and salt tolerance in the melon has not been investigated.

Recently, RNA sequencing (RNA-Seq), using high-throughput sequencing technology, has been widely used to investigate responsive genes or key pathways under abiotic or biotic stress in several plant species, in order to reveal response mechanisms to specific stimuli. For example, salt-stress-regulated genes and their corresponding pathways have been investigated in Brassica napus (Yong et al., 2014), Petunia (Villarino et al., 2014), and Limonium bicolor (Yuan et al., 2015). Data obtained by RNA-Seq have shed light on the bioengineering of active salt secretion capacities in crops. However, to the best of our knowledge, a leaf transcriptome analysis of the salt-tolerant capacity of melon species has not been performed. The release of the complete melon genome sequence (Garcia-Mas et al., 2012) facilitates the study of gene expression patterns without de novo assembly. Hence, high-throughput RNA-Seq provides an opportunity to study muskmelon functional genomics, and to learn the specific molecular mechanisms underlying salt tolerance in the muskmelon.

A previous study of two muskmelon cultivars ('Bing XueCui' and 'Yu Lu') revealed that they exhibited different physiological characteristics under salt stress (Zhu et al., 2010), but the molecular mechanisms underlying the different physiological traits of the two cultivars remain unclear. The objective of this study was to integrate our knowledge of these cultivars' physiological characteristics with a genome-wide transcriptome analysis to understand the molecular basis of genetic variation between salt-sensitive and salt-tolerant melon genotypes under salt stress. A comparison of the leaf transcriptomes showed that a number of varietyspecific responsive genes were related to salt tolerance in the melon. This study provides the first global transcriptome profile of melon leaves, and provides insights into the molecular mechanisms of two melon cultivars in response to salt stress.

\section{MATERIAL AND METHODS}

\section{Plant materials and treatments}

Two Chinese muskmelon cultivars, 'Bing XueCui' (BXC) and 'Yu Lu' (YL), were used. After accelerating germination for 1 day at $30^{\circ} \mathrm{C}$ in a thermostatically controlled incubator, seeds were sown into plugs that contained a mixture of peat, perlite, and slag $(1: 1: 1, \mathrm{v} / \mathrm{v} / \mathrm{v})$, and grown in a glass greenhouse with day/night temperatures of $28^{\circ} / 24^{\circ} \mathrm{C}$, a 14-h photoperiod (lighting level greater than 7000 lux), and a relative humidity of $60 \pm 10 \%$. Seedlings were irrigated using half-strength Hoagland's nutrient solution. Phenotypically uniform plants at the three-leaf stage were selected from each cultivar and transferred to garden pots. After a 3-day colonization period, half-strength Hoagland's solution was used to water the control plants, while the treatment plants were watered with $75 \mathrm{mM}$ increments of $\mathrm{NaCl}$ dissolved in halfstrength Hoagland's solution, to final concentrations of 150 and $300 \mathrm{mM} \mathrm{NaCl}$. The osmolality of each solution was adjusted to the same level using mannitol. The electrical conductivity of the pot percolation solution was monitored daily and corrected by irrigation. During the

Genetics and Molecular Research 15 (3): gmr.15038738 
experiment, the electrical conductivity values were adjusted to salinity levels close to the targeted concentrations of the irrigation solutions of the control (salt-free, $0.5 \mathrm{X}$ Hoagland) and salt-treated plants ( 150 and $300 \mathrm{mM} \mathrm{NaCl}, 0.5 \mathrm{X}$ Hoagland). Three destructive samplings (six plants per genotype per treatment) were conducted at $1 \mathrm{~h}, 24 \mathrm{~h}$, and 7 days after reaching the final saline concentration. Early mature leaves at positions three and four from the apex were collected and stored at $-80^{\circ} \mathrm{C}$ for analysis.

\section{Measurements of $\mathrm{Na}^{+}, \mathrm{K}^{+}$, and $\mathrm{Cl}^{-}$fluxes using NMT}

Three phenotypically uniform, individual melon seedlings from each treatment were used for ion flux measurements after 9 days. Only the 4th leaves were used for obtaining two samples from the abaxial epidermal segments symmetrically along the main leaf axis. Surface hairs were abraded with a brush, and the samples were rinsed quickly and thoroughly in an assay solution comprising $0.1 \mathrm{mM} \mathrm{KCl}, 0.1 \mathrm{mM} \mathrm{CaCl}, 0.1 \mathrm{mM} \mathrm{NaCl}, 0.3 \mathrm{mM}$ anhydrous morpholine ethyl sulfonic acid, and Tris base, $\mathrm{pH}$ 6.15. To minimize ion leakage effects after peeling, particularly from the surfaces of long-term-stressed tissues, the rinsed epidermal fragments were immediately immersed in $3 \mathrm{~mL}$ assay solution for 5-10 min prior to performing the NMT flux experiments. Net $\mathrm{Na}^{+}, \mathrm{K}^{+}$, and $\mathrm{Cl}^{-}$flux measurements in the guard cells, with microelectrodes positioned $1 \pm 0.5 \mu \mathrm{M}$ above the stomatal zone of the epidermal strips, were analyzed by the YoungerUSA (Amherst, MA, USA) NMT Research Service Center (Xuyue, Beijing) using ASET 2.0 (Science Wares Inc., Falmouth, MA, USA) and the iFluxes 1.0 (YoungerUSA) software.

\section{Physiological index measurements}

The net photosynthesis rate (Pn), transpiration rate (EVAP), and stomatal conductivity (gs) were measured in each variety under clear-sky conditions during salt stress using a portable photosynthesis machine (CIRAS-2; PP Systems, Amesbury, MA, USA). After harvesting, the leaves, stems, and roots were separated and washed quickly with distilled water and the fresh weight determined. After drying for $48 \mathrm{~h}$ in an oven at $80^{\circ} \mathrm{C}$, the dry weight was measured. The height of the plants was determined using a ruler. Six plants were collected from each group $(0,150$, and $300 \mathrm{mM} \mathrm{NaCl})$ from each cultivar, after being treated for 1 week for the above indicator measurements.

\section{RNA extraction, library preparation, and sequencing}

Leaves of both cultivars were harvested at $1 \mathrm{~h}, 25 \mathrm{~h}$, and 1 week after the initiation of the 300-mM NaCl treatment. Total RNA was later isolated using TRIzol reagent (Invitrogen, Waltham, MA, USA). RNA concentrations were determined using a NanoDrop ${ }^{\mathrm{TM}} 1000$ Spectrophotometer (Thermo Scientific, Waltham, MA, USA), and the quality of each RNA sample was evaluated using an Agilent 2100 Bioanalyzer (Agilent Technologies, Santa Clara, CA, USA).

A cDNA library was constructed using an RNA-Seq assay for paired-end transcriptome sequencing. The library construction and sequencing were performed by Shanghai Hanyu Bio-Tech (PuDong, Shanghai, China). The library was loaded onto the channels of an Illumina $\mathrm{HiSeq}^{\mathrm{TM}} 2000$ instrument for 2-Gb in-depth sequencing, which was used to obtain detailed information on gene expression. The datasets are available at the National Center

Genetics and Molecular Research 15 (3): gmr.15038738 
for Biotechnology Information (NCBI) Gene Expression Omnibus database (http://www.ncbi. nlm.nih.gov/geo/) under the accession number GSE73380.

\section{Processing of RNA-Seq data}

Clean reads were obtained from the raw data by filtering out adaptor-only and lowquality reads (reads containing less than $20 \%$ bases with a Q-value $<20$ ). The clean reads were then mapped to the C. melo L. genome sequences using TopHat2 (https://ccb.jhu.edu/ software/tophat/index.shtml). The mapped reads were assembled into transcripts and their abundances calculated using Cufflinks (http://cole-trapnell-lab.github.io/cufflinks/). The gene expression levels were measured in fragments per kb of transcript per million mapped reads.

\section{Identification of differentially expressed genes (DEGs)}

The differential expression of a gene was calculated by Cuffdiff (Cufflinks) based on transcript abundances. Genes with a $\mathrm{P}$ value $<0.05$ and at least a 2 -fold change (either up- or downregulation) were considered differentially expressed between the salt stress and control samples of each cultivar.

\section{Function and pathway-enrichment analyses}

Gene Ontology (GO) terms were assigned to the identified genes with E-values less than $10^{-6}$ by Blast2GO (https://www.blast2go.com/) using the NCBI non-redundant database (Aparicio et al., 2006). A functional enrichment of the gene sets was performed using the Fisher exact test implemented in Blast2GO.

The enrichment of Kyoto Encyclopedia of Genes and Genomes (KEGG) pathways with stress-responsive gene sets was calculated using the cumulative hypergeometric distribution. The probability of a KEGG pathway was given by P, as follows:

$$
P\left(x^{\prime} \mid K, M, N\right)=1-\sum_{0}^{x-1} \frac{\left(\begin{array}{c}
M \\
x
\end{array}\right)\left(\begin{array}{l}
N-M \\
K-x
\end{array}\right)}{\left(\begin{array}{l}
N \\
K
\end{array}\right)}
$$

Equation 1

where $N$ is the total number of muskmelon genes, $M$ is the number of genes annotated to a particular KEGG pathway, $K$ is the number of DEGs, and $x$ is the number of DEGs annotated to a particular KEGG pathway. We identified significantly $(\mathrm{P}<0.05)$ enriched KEGG pathways that contained DEGs.

\section{Verification of DEGs by real-time quantitative reverse transcription-polymerase chain reaction (qRT-PCR)}

The total-RNA samples were reverse transcribed using a First-Strand cDNA Synthesis Kit (Takara, Japan) and amplified using a $\mathrm{SYBR}^{\circledR}$ Green Mix Kit (Takara) by real-time qRTPCR on an FTC-3000 qPCR system (Funglyn Biotech, Shanghai, China). Twelve genes were selected to confirm their expression responses to salt stress by real-time qRT-PCR. Genespecific primers were designed by the Primer Premier software based on the obtained partial

Genetics and Molecular Research 15 (3): gmr.15038738 
sequences of the genes listed in Table S1. The amplification program included $30 \mathrm{~s}$ for the activation of the polymerase at $95^{\circ} \mathrm{C}$, followed by 45 cycles at $95^{\circ} \mathrm{C}$ for $5 \mathrm{~s}, 57^{\circ} \mathrm{C}$ for $30 \mathrm{~s}$, and $72^{\circ} \mathrm{C}$ for $30 \mathrm{~s}$. Standard curves were derived from a series of five 30 -fold dilutions of the cDNA template. The expression levels of the selected cDNAs were determined relative to an actin reference gene from four independent biological replicates. The relative quantification method $\left(2^{\Delta \Delta \mathrm{Ct}}\right)$ was used to evaluate the quantitative variation between the treatments. The expression level of each target gene was normalized and calculated to the level of the actin reference gene.

\section{Statistical analysis}

The results are reported as means \pm SE. Comparisons of the mean values were performed using the Fisher least significant difference test in SAS 9.1 (SAS Institute Inc., Cary, NC, USA); the level of significance was set at $\mathrm{P}<0.05$. Pearson's correlation coefficients were calculated using SigmaPlot ${ }^{\circledR} 11$.

\section{RESULTS}

\section{Phenotypes and biomass values of the two melon cultivars}

The phenotypes of the BXC and YL plants are shown in Figure 1A. Salinity depressed the performances of the two cultivars, which was particularly evident at the highest $\mathrm{NaCl}$ concentration. Salt-stress damage, such as necrosis and wilting, appeared on YL leaves after approximately 1 week of the $300-\mathrm{mM}$ salt treatment. Similar damage, but on relatively fewer leaves, was observed on BXC.

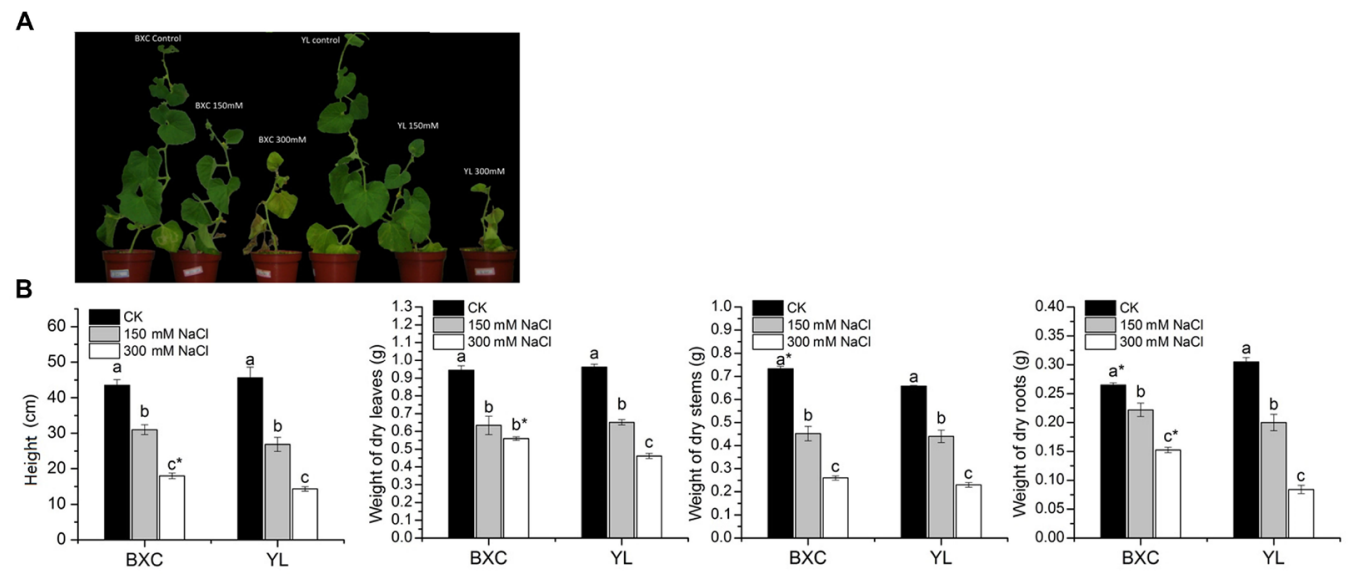

Figure 1. Effect of salt stress on the phenotype, height, and biomass of two muskmelon seedlings. A. The growth of both salt-treated plants was severely restricted compared to that of the control plants. Leaf damage caused by salinity stress appeared on BXC and YL leaves, particularly at $300 \mathrm{mM} \mathrm{NaCl}$. B. Height and leaf, stem, and root dry weights of 1-week-old salt-stressed and control plants. Data are reported as means $\pm \mathrm{SE}(\mathrm{N}=6)$.

Genetics and Molecular Research 15 (3): gmr.15038738 
In general, increasing the $\mathrm{NaCl}$ concentration reduced seedling growth and the biomass (i.e., dry weight) of each individual tissue (leaf, stem, and root) in both cultivars. After 1 week of the $300-\mathrm{mM} \mathrm{NaCl}$ treatment, the heights of $\mathrm{BXC}$ and $\mathrm{YL}$ were 58.6 and $70.5 \%$ of the control plants, respectively (Figure 1B). The dry weights of BXC leaves and roots were greater than those of YL over the study period ( $\mathrm{P}<0.05$ ), particularly at $300 \mathrm{mM} \mathrm{NaCl}$ (Figure 1B).

\section{$\mathrm{Na}^{+}, \mathrm{K}^{+}$, and $\mathrm{Cl}^{-}$fluxes in guard cells}

The NMT measurements in the stomatal zone $(1 \pm 0.5 \mu \mathrm{m}$ above the pore $)$ revealed a similar trend in terms of $\mathrm{Na}^{+}, \mathrm{K}^{+}$, and $\mathrm{Cl}^{-}$fluxes in both cultivars when exposed to high-salinity stress $(300 \mathrm{mM})$. Significant increases in $\mathrm{Na}^{+}$and $\mathrm{K}^{+}$effluxes, and the $\mathrm{Cl}^{-}$influx, were found in both high-salt-treated cultivars in comparison with plants under non-saline conditions. YL stomata retained a higher capacity to exclude $\mathrm{Na}^{+}$than did $\mathrm{BXC}$ stomata.

However, at $150 \mathrm{mM} \mathrm{NaCl}$, no differences in the net fluxes of these three ions were observed between salt-treated and control BXC plants. However, $\mathrm{Na}^{+}$and $\mathrm{K}^{+}$exclusions were detected in YL. There was little change in the net $\mathrm{Cl}^{-}$flux in $\mathrm{YL}$ at this lower saline level (Figure 2).
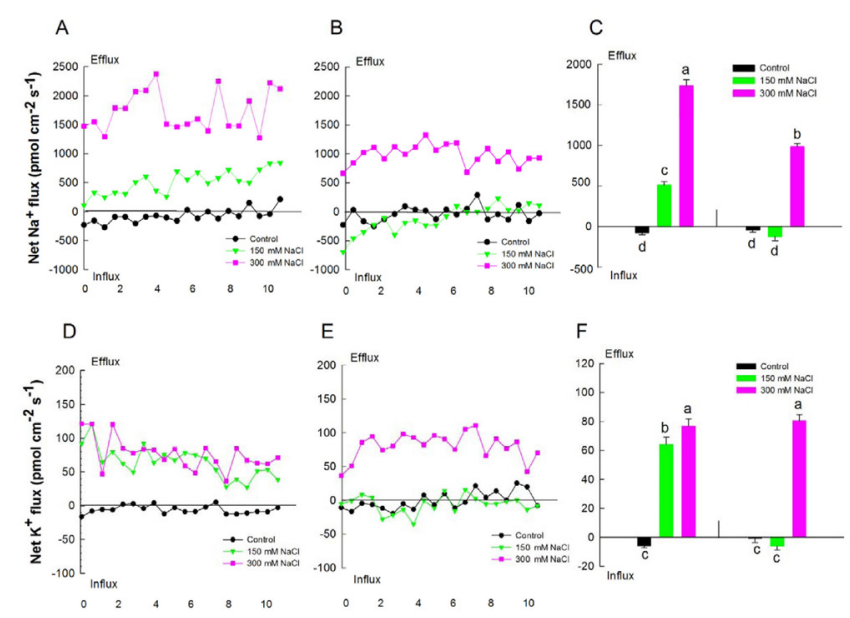

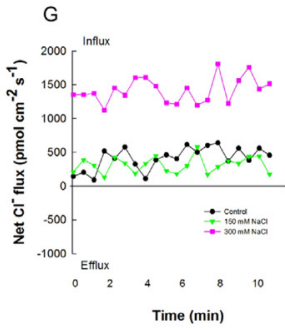

YL

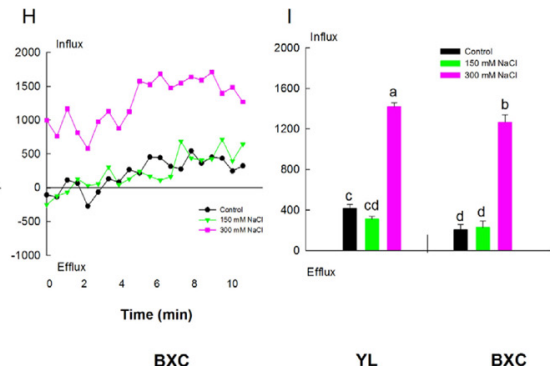

Figure 2. Regulation of ionic fluxes in the stomatal zones of two muskmelon cultivars in response to salt stress. The continuous net $\mathrm{Na}^{+}, \mathrm{K}^{+}$, and $\mathrm{Cl}^{-}$fluxes of two muskmelon cultivars grown at 0 (black), 150 (green), and $300 \mathrm{mM}$ $\mathrm{NaCl}$ (pink) over $10 \mathrm{~min}(\mathbf{A}, \mathbf{B}, \mathbf{D}, \mathbf{E}, \mathbf{G}$, and $\mathbf{H})$; each point represents the mean of six individual measurements. The mean fluxes of $\mathrm{Na}^{+}, \mathrm{K}^{+}$, and $\mathrm{Cl}^{-}$within the recording period are shown in $\mathbf{C}, \mathbf{F}$, and $\mathbf{I}$, respectively. Columns marked with different letters indicate a significant difference at $\mathrm{P}<0.05$. 


\section{Photosynthetic characteristics}

The saline applications caused reductions in the EVAP, Pn, and gs of both muskmelon cultivars. However, based on these three indices, BXC was significantly less affected by the $150-\mathrm{mM} \mathrm{NaCl}$ treatment than YL. YL exhibited similarly reduced levels of these variables at the two saline concentrations, indicating that this cultivar was detrimentally affected at the 150-mM NaCl level (Figure 3).

A

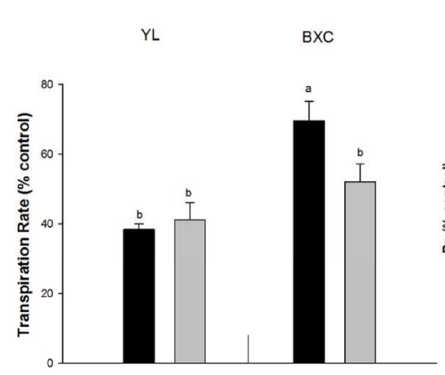

B

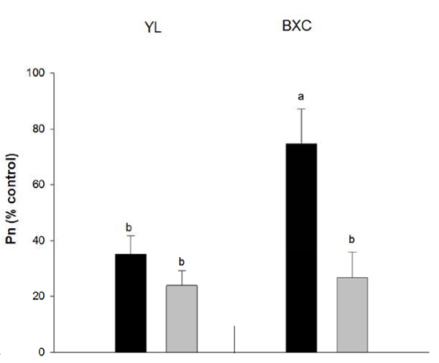

C

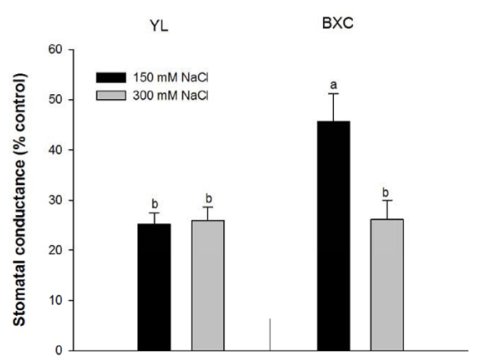

Figure 3. Effect of salinity on the relative change (\% control) in transpiration rate (A), net photosynthesis rate $(\mathrm{Pn})$ (B), and stomatal conductance (C) of two muskmelon seedlings. Error bars indicate the standard error of the mean (SE) of six measurements. Columns labeled with different letters at the top denote a significant difference at $\mathrm{P}<0.05$.

\section{Global analysis of gene expression levels in the two melon cultivars}

Four cDNA libraries were constructed using total RNA from BXC and YL under both control and salt-stress conditions $(300 \mathrm{mM} \mathrm{NaCl})$. Each library was processed on the Illumina platform. After removing low-quality reads, the total number of clean read pairs ranged from 4.8 to $14 \mathrm{M}$. The high-quality reads from each library were then aligned to the $C$. melo $\mathrm{L}$. genome, and $79-83 \%$ of the reads were mapped to the reference database, comprising $\sim 19$ $24 \mathrm{~K}$ mapped genes (Table 1). These genes were used for further analyses. Under normal conditions, the expression levels of 522 genes were higher and 364 genes were lower in BXC than in YL; after salt stress, the expression levels of 1204 genes were higher and those of 328 genes were lower in BXC than in YL (Figure S1). Compared with the control, genes in both cultivars were divided into two libraries, with blue and red dots indicating significant and nonsignificant changes, respectively (Figure 4A). A total of 1171 DEGs were found in response to salt stress in BXC, 572 of which were upregulated and 599 downregulated. In YL, 1487 genes were identified as salt-stress-responsive DEGs, with 538 being upregulated and 949 downregulated (Figure 4B). Twelve genes with differential expression patterns were selected for real-time qRT-PCR analysis to verify the reliability of the DEGs inferred from the RNASeq. The correlation between the RNA-Seq and qRT-PCR data was evaluated using foldchange measurements. The real-time qRT-PCR results were significantly correlated $(\mathrm{r}=0.81)$ with the RNA-Seq data (Figure 4C), demonstrating the reliability of the RNA-Seq results.

A large group of cultivar-specific responsive genes existed under salt stress, with 560 in BXC and 876 in YL. The number of salt-stress-induced genes was significantly higher than that of salt-stress-inhibited genes in BXC, whereas the opposite was the case in YL. A complete list of DEGs is provided in Table $\mathbf{S 2}$.

Genetics and Molecular Research 15 (3): gmr.15038738 
Table 1. Summary of sequencing data.

\begin{tabular}{c|c|c|c|c|c}
\hline Library & $\begin{array}{c}\text { No. of raw data } \\
\text { pairs }\end{array}$ & $\begin{array}{c}\text { No. of high-quality clean } \\
\text { read pairs }\end{array}$ & $\begin{array}{c}\text { No. of clean } \\
\text { mapped reads }\end{array}$ & $\begin{array}{c}\text { Reads mapped at high } \\
\text { quality (\%) }\end{array}$ & No. of mapped genes \\
\hline B0 & $7,545,783$ & $5,967,247$ & $9,932,433$ & 83.22 & 19,856 \\
\hline B2 & $5,866,812$ & $4,800,239$ & $7,842,096$ & 81.68 & 21,423 \\
\hline Y0 & $17,615,882$ & $13,059,581$ & $22,403,164$ & 85.77 & 20,918 \\
\hline Y2 & $17,504,211$ & $14,266,787$ & $22,537,785$ & 78.99 & 23,563 \\
\hline
\end{tabular}

$\mathrm{B} 0$ and $\mathrm{B} 2, \mathrm{BXC}$ under control and 300-mM NaCl conditions, respectively; $\mathrm{Y} 0$ and $\mathrm{Y} 2$, YL under control and 300$\mathrm{mM} \mathrm{NaCl}$ conditions, respectively.

A
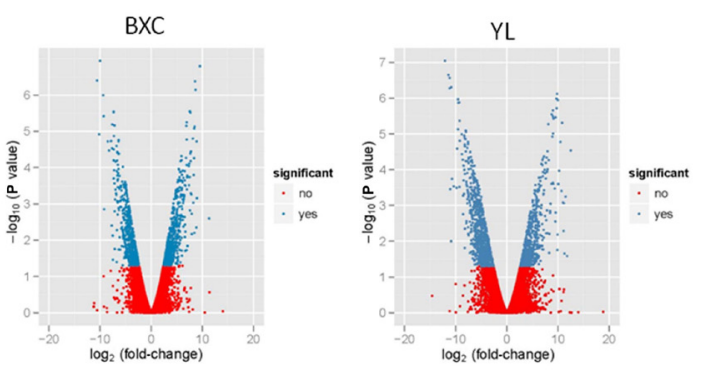

B

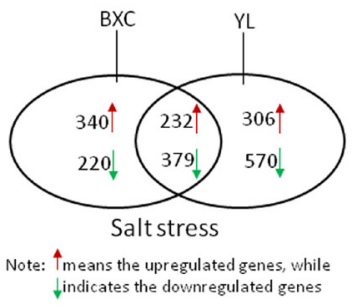

C

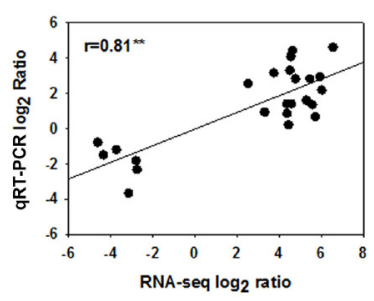

Figure 4. Differentially expressed genes in two muskmelon cultivars. A. Gene expression levels in two libraries. The $\mathrm{x}$-axis represents the fold-change of differentially expressed genes in the library, and the y-axis represents the $\mathrm{P}$ value $\left(\log _{10}\right) . \mathrm{P}<0.05$ and $\log _{2}$ ratio $\geq 1$ or $\leq-1$ were the criteria used to indicate a significant difference in gene expression between treated and untreated samples of BXC (left panel) and YL (right panel). Red dots represent the expression levels of genes that did not change significantly in the library, and blue dots indicate genes that changed significantly. B. Venn diagram of differentially expressed genes under salt stress in BXC and YL. This figure illustrates the number of up- and downregulated genes in BXC and YL after salt stress. C. Correlation analysis of gene expression levels obtained from RNA sequencing and real-time polymerase chain reaction (PCR) data. Twelve genes were selected for PCRs in two libraries (BXC and YL) after salt stress. The expression level of each target gene was normalized and calculated based on the level of the actin reference gene. The values were normalized again based on the expression levels of each gene under control conditions and the data were plotted as $\log _{2}$ ratio (salt stress/control) for each gene.

\section{Functional categories of species-specific responsive genes in the two cultivars}

To identify the functional categories of species-specific salt-responsive genes in the two cultivars, GO was employed to classify sequences annotated by known proteins. Based on the GO functional catalog, of the 1436 species-specific differentially regulated genes, 894 were annotated with known functions (detailed functional categories are shown in Table S3 ). Of the upregulated genes, fewer were from YL than from BXC in almost all of the functional categories (Figure 5A). However, the downregulated genes that were related to various 
biological processes were augmented in YL, and accounted for a larger proportion of the total than those in BXC (Figure 5B). The distribution of enriched GO terms shows that processes such as oxidation-reduction, ion binding, and substrate-specific transporter activity greatly differed between BXC and YL (Table S4), suggesting that the two cultivars have different response mechanisms at the transcriptional level under salt stress.

A

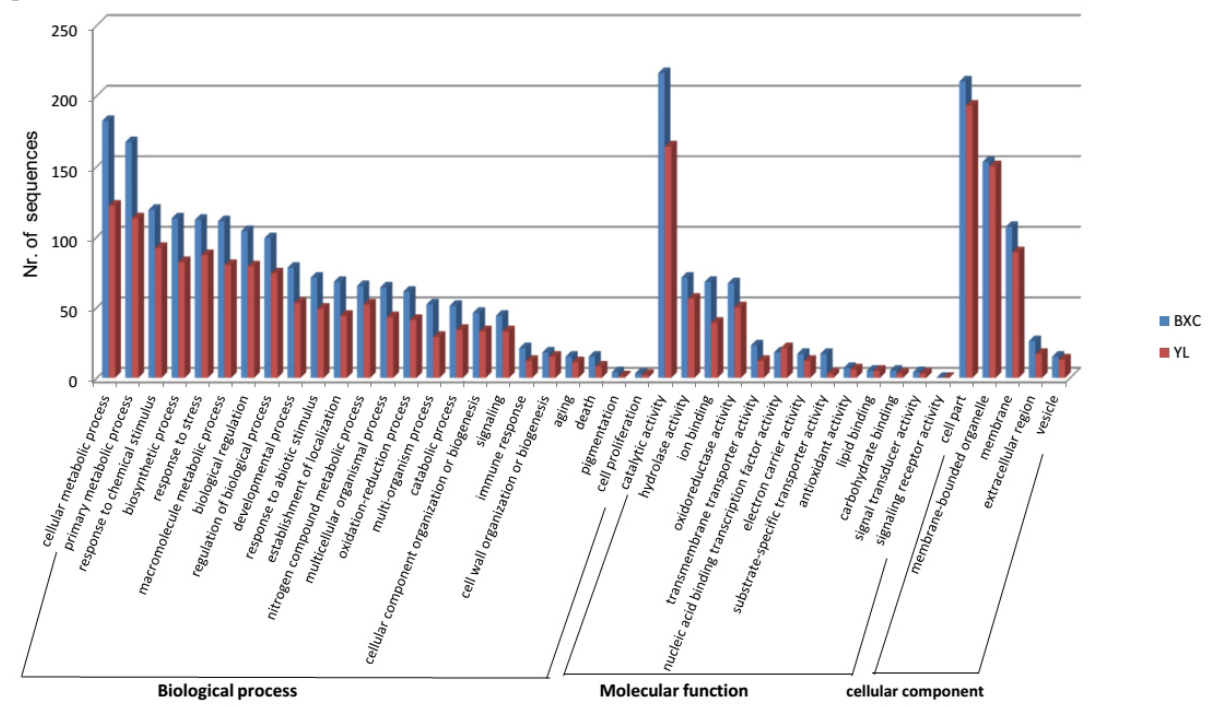

B

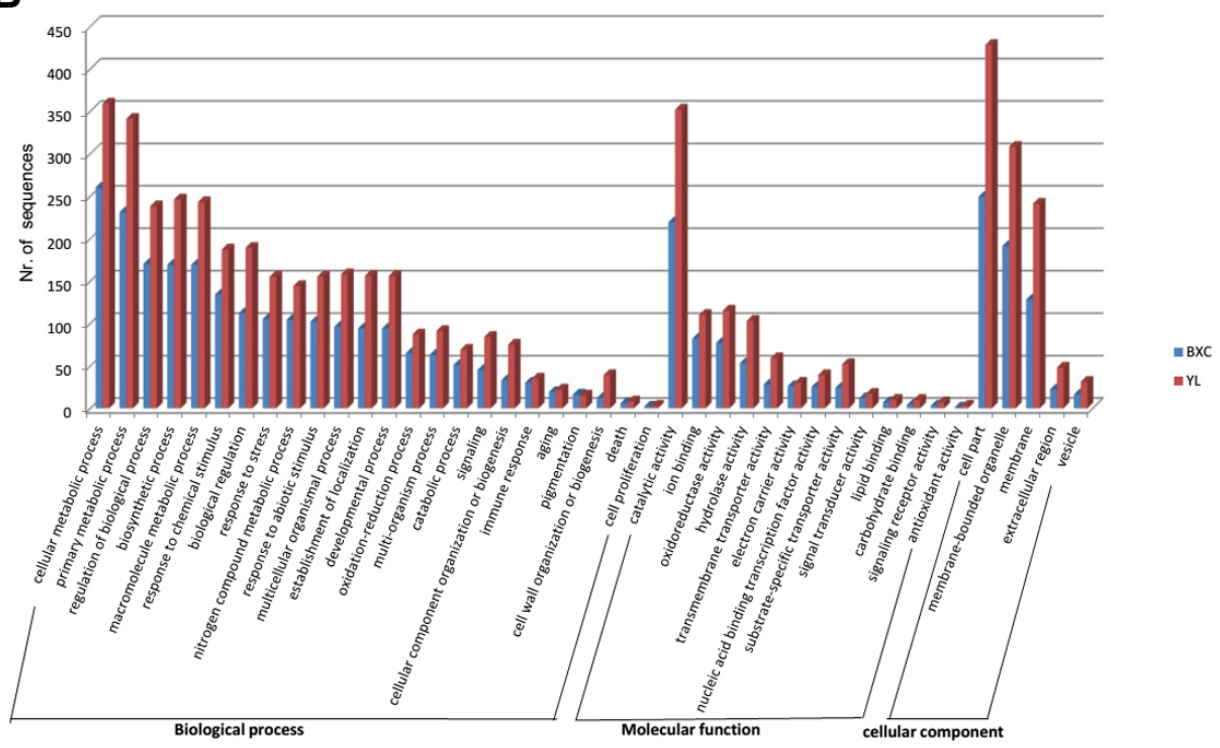

Figure 5. Gene ontology (GO) categories of differentially expressed genes of two muskmelon cultivars. A. Upregulated sequences in BXC and YL. B. Downregulated sequences in BXC and YL. In order to simplify the display, the figure only shows some of the GO categories.

Genetics and Molecular Research 15 (3): gmr.15038738 
In addition to the species-specific regulated genes, there were a large number of overlapping responsive genes between the two cultivars, including 232 upregulated genes and 379 downregulated genes (Figure 4B). A complete list of the common regulated genes is provided in Table S5. Based on the GO functional catalog, 155 of the 232 upregulated genes were assigned GO terms (Figure 5A). In the "Biological Process" category, genes in "response to stimulus" accounted for $15.3 \%$. The "catalytic activity" and "binding" groups within the "Molecular Function" category accounted for more than $35 \%$ of the annotated sequences. In addition, some sequences were involved in other important functional processes, such as "biological regulation", "response to signaling", "transcription factor activity", and "transporter activity". Detailed information is provided in Table S6.

Similar to the functional distribution of the upregulated genes, 127 of the 306 downregulated genes were assigned GO terms (Figure 5B). The 127 downregulated sequences were mainly aggregated into the categories of important biological processes such as "cellular process", "metabolism", "stimulus response", and "biological regulation and binding" (Table S7). In addition, most of these categories contained a greater proportion of downregulated probe sets than upregulated probe sets ( $\underline{\text { Table S8 }}$ ).

\section{Pathway-enrichment analysis of salt-responsive genes}

To understand the biological functions and interactions of the genes, a pathway-based analysis was conducted based on the KEGG pathway database. A pathway-enrichment analysis of the up- and downregulated genes of BXC and YL showed that salt-stress-responsive genes were significantly enriched in $26 \mathrm{KEGG}$ pathways $(\mathrm{P}<0.05$; Table $\mathbf{S 9}$ ). Based on quantitative comparisons of up- and downregulated genes in $\mathrm{BXC}$ and $\mathrm{YL}$, we found several pathways that highlighted the differences between BXC and YL in response to salt stress, including "photosynthesis", "biosynthesis of secondary metabolites", "metabolic pathway", and "plant hormone signal transduction" (Figure 6).

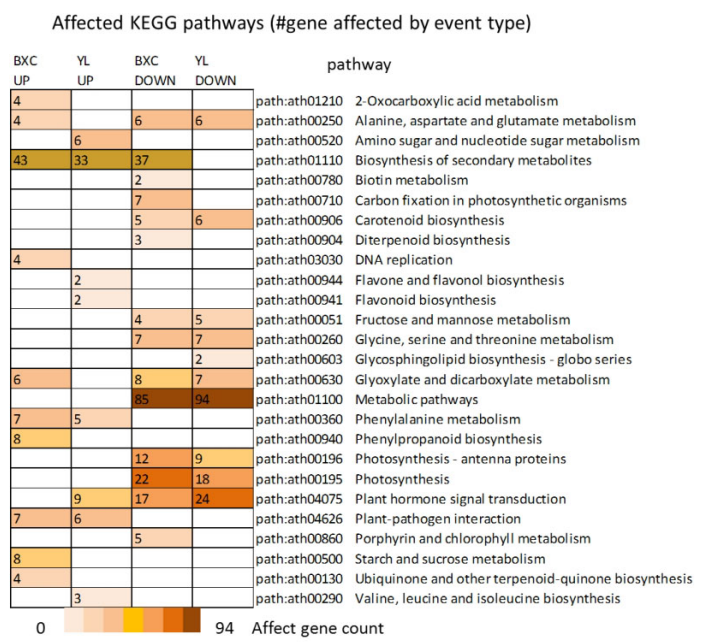

Figure 6. Kyoto Encyclopedia of Genes and Genomes (KEGG) pathways of two muskmelon cultivars. KEGG pathway gene sets enriched with commonly upregulated or downregulated genes under control and high-salt conditions. Each column represents a data count involving the significant pathways. 
The upregulated genes in BXC were significantly enriched in the "starch and sucrose metabolism", "DNA replication", "phenylpropanoid biosynthesis", and "ubiquinone and other terpenoid-quinone biosynthesis" pathways. The categories "carbon fixation in photosynthetic organisms" and "porphyrin and chlorophyll metabolism" contained more downregulated genes in BXC than in YL. An enrichment analysis revealed that the upregulated genes in YL were significantly enriched in the "flavone and flavonol biosynthesis" pathway.

\section{Comparison of 12 genes in the two cultivars in response to salt stress}

Genes that encode transcription factors such as NAC, WRKY, and MYB were analyzed by qRT-PCR (Figure 7A). Both $N A C 1$ and $N A C 2$ responded immediately to salt stress in both cultivars, generally with an upregulated trend. The highest transcription level occurred after 1 week of treatment in both cultivars, and both genes were expressed at higher levels in BXC than in YL. WRKY1 and WRKY2 were upregulated in both cultivars after 1 week of salt treatment, but exhibited different trends over time. Under short-term salt stress $(1$ or $24 \mathrm{~h}$ ), WRKY1 was immediately downregulated, while WRKY2, in general, was upregulated. $M Y B 1$ and $M Y B 2$ were suppressed immediately after $1 \mathrm{~h}$ of salt stress, and were eventually upregulated after 1 week. However, the initial downregulation effect on MYB1 expression was reversed at different times in the two cultivars, with upregulation occurring earlier in BXC than in YL. For MYB2, both cultivars shared the same regulating principle over time, and YL was observed to have a higher induction factor after 1 week.

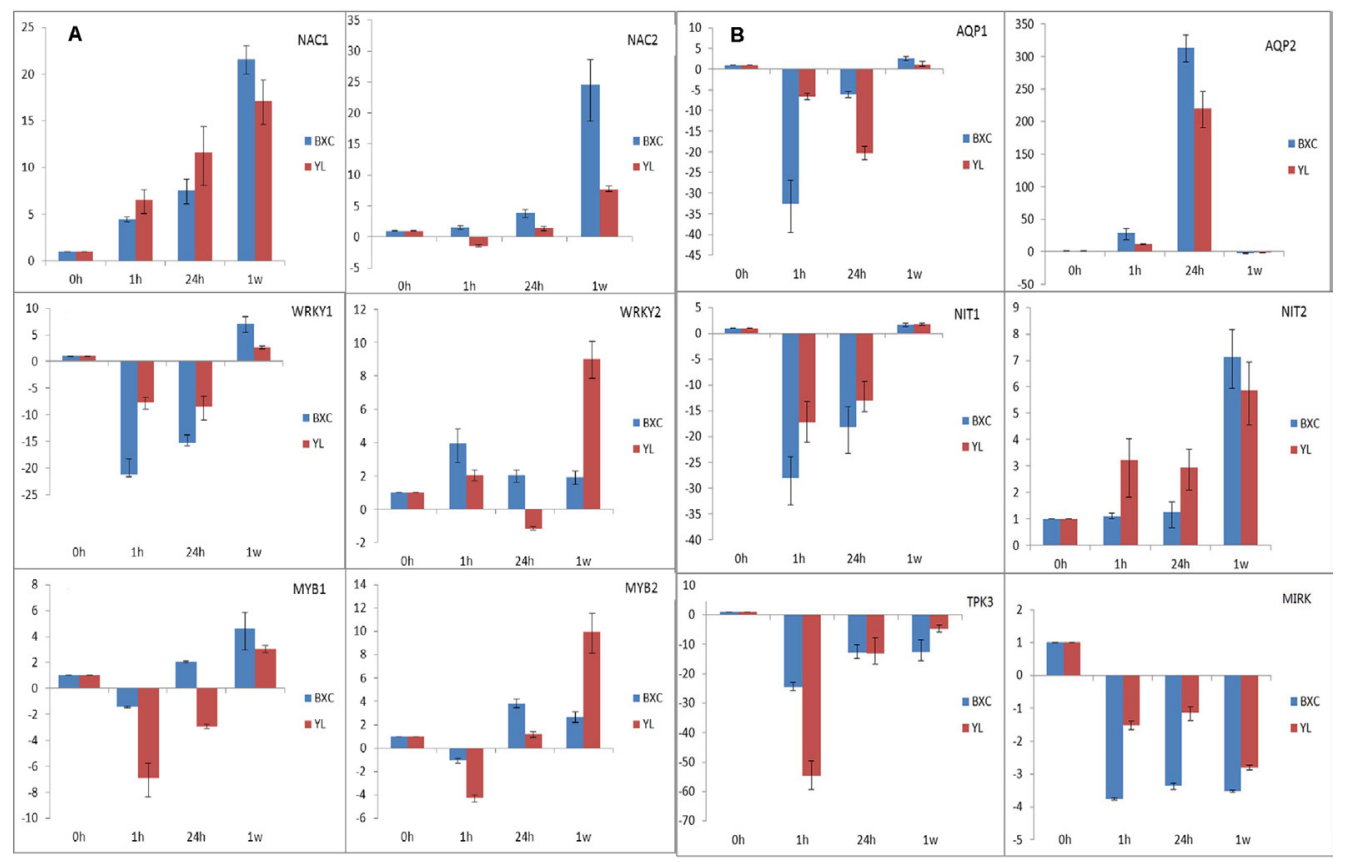

Figure 7. Expression levels of 12 selected genes at three time points ( $1 \mathrm{~h}, 24 \mathrm{~h}$, and 1 week) in BXC and YL. The $\mathrm{y}$-axis represents the expression level relative to the actin reference gene; the relative expression level of each gene in the control treatment $(0 \mathrm{~h})$ was set at 1 . Error bars represent the standard error of the mean (SE) of three independent experiments.

Genetics and Molecular Research 15 (3): gmr.15038738 
Genes that encode water or ionic transporters were studied by qRT-PCR (Figure 7B). The aquaporin genes $A Q P 1$ and $A Q P 2$ were affected by salt stress, and exhibited the same expression-related trends in the two cultivars. However, differences in the expression levels of these two genes were observed under short-term stress (1 and $24 \mathrm{~h}$ ). Two nitrate transporters, nitrate transporter 1.5-like (NIT1.5) and nitrate transporter 1.2-like (NIT1.2), were both induced after 1 week of treatment. In both cultivars, NIT1.5 was downregulated in the short term; however, NIT1.2 was upregulated during the entire period. Two additional genes, TPK3 and $M I R K$, which encode K channels, were also analyzed; we found that both genes, in both cultivars, were downregulated during the entire treatment period. The extent of the downregulation of the genes was more readily distinguished between the two cultivars over the short period $(1 \mathrm{~h})$ than over longer periods. The sequences of the 12 genes are presented in Table S10.

\section{DISCUSSION}

\section{BXC was more tolerant to salt stress than YL during the early stage}

Several melon cultivars that differ in salt tolerance have been studied (Mangal et al., 1988). We found that in response to salt stress, more damage occurred to the leaves of YL than to those of $\mathrm{BXC}$, and was accompanied by a greater reduction in biomass, which is in line with the negative correlation found between relative biomass and damage in barley (Wu et al., 2015). This suggests that BXC had a better phenotypic performance than YL under salt stress. In addition, BXC was significantly less affected by salt stress than YL in terms of EVAP, Pn, and gs, indicating that BXC still maintained a relatively greater capability for photosynthesis (Tester and Davenport, 2003), particularly at $150 \mathrm{mM} \mathrm{NaCl}$.

Guard cells gate stomatal pores in order to balance gas exchange (mainly $\mathrm{CO}_{2}$ ) and transpiration, by modulating ionic activity in their plasma membranes (Laanemets et al., 2013). At low saline levels in BXC, there were no significant differences in ionic fluxes between salt-treated plants and control plants. However, greater $\mathrm{Na}^{+}$and $\mathrm{K}^{+}$exclusion was detected in the guard cell zone in YL salt-treated plants, indicating that the YL plants were subject to the detrimental effects of high salinity. The $\mathrm{Na}^{+}$efflux (but not the $\mathrm{K}^{+}$efflux) under saline conditions was significantly, negatively correlated with the EVAPs and shoot biomasses $[\mathrm{r}=$ -0.91 and -0.99 , respectively (data not shown)], suggesting that the high EVAP in BXC under salt stress was caused by the lower $\mathrm{Na}^{+}$efflux. Therefore, $\mathrm{Na}^{+}$exclusion may be the key ionic indicator for salt stress in melon species, and BXC is more tolerant of high salinity than YL, based on the physiological indices.

\section{Transcriptome analyses of the regulation of reactive oxygen species (ROS)- responsive genes in the two melon cultivars}

Under salt stress, ROS accumulates, resulting in damage to membranes and related biological macromolecules. Plants have developed several strategies to minimize the oxidative damage of ROS using antioxidant enzyme systems (Sandalio et al., 2013). Peroxidases (PODs), which are antioxidative enzymes, play key roles in cellular ROS detoxification under salt stress (de Azevedo Neto et al., 2006). In our study, genes that encode PODs and a POD precursor were induced by salt stress in both cultivars, so they could be considered strong indicators of salt stress in melon species.

Genetics and Molecular Research 15 (3): gmr.15038738 
Another group of salt-stress-responsive genes are glutathione S-transferases (GSTs), which promote cell survival under oxidative stress. Plant GSTs play roles in herbicide detoxification, and also act as glutathione PODs by protecting cells from oxygen toxicity and suppressing apoptosis. GST gene expression that promotes a greater level of salt tolerance has been verified in vivo in transgenic Arabidopsis (Qi et al., 2010). In our study, genes that are related to GSTs were induced in BXC but not in YL (Table S11). This upregulated expression in $\mathrm{BXC}$ may help to protect cells from the oxygen toxicity that increases in leaves under salt stress, thereby enhancing the growth of BXC seedlings.

However, glutathione reductase (GR) transcription was specifically induced in YL. In the ascorbate-glutathione cycle, GR contributes to the defense against ROS by maintaining glutathione in its reduced state (Gill and Tuteja, 2010). A genotype-specific induction of the ROS-scavenging pathway may lead to a better tolerance of abiotic stressors. Therefore, the induction of GR genes in YL could be a specific response linked to salt resistance in this cultivar. These results suggest an underlying difference in the protection mechanisms against oxidative damage caused by salt stress between the two cultivars.

\section{Transcriptome analyses of salt-responsive genes that encode water and ionic transporters in the two melon cultivars}

As vital proteins in plant growth and development, abundant levels of aquaporins exist in most living organisms, and are involved in many physiological processes including water uptake and transportation, micronutrient uptake, reproduction, and photosynthesis (Heinen et al., 2009). Under saline conditions, a rapid increase in the intracellular salt concentration causes a rapid outflow of water from the cells via aquaporins, thereby leading to an osmotic effect (Allakhverdiev et al., 2000). For example, aquaporin-deficient cells of Synechocystis are unable to efficiently regulate the cytoplasmic volume under salt stress (Sinetova et al., 2015), and overexpressing some aquaporin genes enhances the salt tolerance of transgenic plants (Sun et al., 2015). In our study, $A Q P 1$ and $A Q P 2$ were affected by salinity over time in both cultivars. As $A Q P 1$ expression decreased over the short-term, $A Q P 2$ expression increased (Figure 7B). This opposite regulation in both cultivars suggests that a common supplementary mechanism controlled by $A Q P 2$ in melon species exists, which maintains membrane water permeability and cellular osmotic balance under salt stress.

Genes that encode nitrate, ammonium, and amino acid transporter proteins were mostly downregulated under long-term salinity in both BXC and YL (Table S11), except for two nitrate transporters, NIT1.5 (XM_008467720) and NIT1.2 (XM_008454950) (Figure 7B) that might be involved in maintaining nitrate transportation (Lin et al., 2008 ). In general, the exposure of plants to high-salt stress reduces the $\mathrm{K}^{+}$level in the whole plant or in individual tissues (Khan et al., 2000). We found that TPK3 and MIRK were downregulated in the leaves after salt stress (Figure 7B). Both genes exhibit $\mathrm{K}^{+}$-selective channel activity (Zhang et al., 2011; Carraretto et al., 2013). TPK3silenced Arabidopsis plants exhibit reduced growth and altered thylakoid membrane organization, resulting in reduced $\mathrm{CO}_{2}$ assimilation and a reduced non-photochemical dissipation of excess absorbed light (Carraretto et al., 2013). MIRK, which is located in guard cells (Zhang et al., 2011), may be involved in stomatal movement (Wang et al., 2013). The extent of the downregulation of both genes was more distinguishable between the two cultivars over the short duration $(1 \mathrm{~h})$ than over longer periods. The potential utility of these two genes as rapid molecular markers to screen for the salinity-tolerant capacities of different melon cultivars needs to be further investigated.

Genetics and Molecular Research 15 (3): gmr.15038738 


\section{Transcriptome analyses of salt-stress-responsive transcription factors in the two melon cultivars}

Transcription factors that control the expression levels of stress-responsive genes are important in regulating plant responses to a range of abiotic and biotic stressors (Singh et al., 2002). We focused on six genes that successively encode NAC, WRKY, and MYB transcription factors.

NAC transcription factors play important roles in stress responses and plant development (Kawaura et al., 2008). The overexpression of some NAC genes increases the salinity tolerance of transgenic plants (Zheng et al., 2009). $N A C 1$ and $N A C 2$ were upregulated under salt stress in both cultivars, with both, particularly $N A C 2$, expressed at higher levels in the salt-tolerant $\mathrm{BXC}$ cultivar than in the salt-sensitive $\mathrm{YL}$ cultivar, indicating that $N A C 2$ is potentially useful for engineering salt-tolerant melons.

WRKY transcription factors are indispensable for transcriptional reprogramming, and are often associated with plant stress responses; auto- or cross-regulation among WRKY genes has been extensively recorded (Chen et al., 2012). In general, WRKY genes exhibit immediateearly regulatory behavior (Hara et al., 2000), which may ensure the success of stress-signaling cascades that activate adaptive responses and regulate stress-related genes, eventually resulting in stress tolerance (Chen et al., 2012). In our study, immediate differences in the expression levels of both WRKY genes, either the downregulation of WRKY1 or the upregulation of WRKY2, were also observed after $1 \mathrm{~h}$ of salinity stress in both cultivars (Figure 7A), indicating that WRKY family members may act as regulatory factors involved in salt-stress responses through complex mechanisms. Interestingly, variations in both genes' expression levels were much more noticeable in the salt-tolerant cultivar BXC than in YL. This may be related to a rapid recognition of salt stress, which activates adaptive responses and regulates stress-related genes in order to tolerate a high-salt environment (Chinnusamy et al., 2004).

Abundant genes that encode MYB transcription factors respond to salinity stress by up- or downregulation (Kawaura et al., 2008). In the present study, six MYB proteins were upregulated and four downregulated after salt stress. MYB1 and MYB2 were investigated over time in the $300-\mathrm{mM}$ salt-stress treatment (Figure 7A). Both genes were suppressed immediately after $1 \mathrm{~h}$ of salt stress, and were eventually upregulated after 1 week. However, the reversal in MYB1 expression occurred at different times in the two cultivars, with upregulation appearing earlier in BXC than in YL, which may be advantageous when preparing plants for stress (Mattana et al., 2005). For MYB2, both cultivars followed the same trend, by either upregulating or downregulating over time, but eventually there was higher induction in YL, suggesting an important role for this gene in the stress defenses of the salt-sensitive cultivar.

\section{CONCLUSIONS}

In summary, BXC performed better under salt stress than YL in terms of biomass and photosynthesis. A transcriptome comparison of the two melon cultivars showed that 1171 genes responded to salt stress in BXC while 1487 were identified as salt-stress-responsive in YL. A significant, positive correlation between the real-time qRT-PCR results and the RNASeq data for 12 genes confirmed the reliability of the latter. We found several pathways that differed between the two cultivars in response to salt stress, including "photosynthesis", "biosynthesis of secondary metabolites", "metabolic pathways", and "plant hormone signal

Genetics and Molecular Research 15 (3): gmr.15038738 
transduction". Our study provides molecular information for further research on salt-tolerant mechanisms in the melon.

\section{Conflicts of interest}

The authors declare no conflict of interest.

\section{ACKNOWLEDGMENTS}

We thank the YoungerUSA (Xuye, Beijing) NMT Service Center for their technical assistance, and Sergey Shabala of the University of Tasmania for suggestions on the ion flux experiments. Research supported by the National Nature Science Foundation of China (\#31372079) and the Nature Science Foundation of Shanghai (\#13ZR1422400).

\section{REFERENCES}

Allakhverdiev SI, Sakamoto A, Nishiyama Y and Murata N (2000). Inactivation of photosystems I and II in response to osmotic stress in Synechococcus. Contribution of water channels. Plant Physiol. 122: 1201-1208. http://dx.doi. org/10.1104/pp.122.4.1201

Aparicio G, Götz S, Conesa A, Segrelles D, et al. (2006). Blast2GO goes grid: developing a grid-enabled prototype for functional genomics analysis. Stud. Health Technol. Inform. 120: 194-204.

Carraretto L, Formentin E, Teardo E, Checchetto V, et al. (2013). A thylakoid-located two-pore $\mathrm{K}^{+}$channel controls photosynthetic light utilization in plants. Science 342: 114-118. http://dx.doi.org/10.1126/science. 1242113

Chen L, Song Y, Li S, Zhang L, et al. (2012). The role of WRKY transcription factors in plant abiotic stresses. Biochim. Biophys. Acta 1819: 120-128. http://dx.doi.org/10.1016/j.bbagrm.2011.09.002

Chinnusamy V, Schumaker K and Zhu JK (2004). Molecular genetic perspectives on cross-talk and specificity in abiotic stress signalling in plants. J. Exp. Bot. 55: 225-236. http://dx.doi.org/10.1093/jxb/erh005

Cuin TA, Betts SA, Chalmandrier R and Shabala S (2008). A root's ability to retain $\mathrm{K}^{+}$correlates with salt tolerance in wheat. J. Exp. Bot. 59: 2697-2706. http://dx.doi.org/10.1093/jxb/ern128

de Azevedo Neto AD, Prisco JT, Enéas-Filho J, de Abreu CEB, et al. (2006). Effect of salt stress on antioxidative enzymes and lipid peroxidation in leaves and roots of salt-tolerant and salt-sensitive maize genotypes. Environ. Exp. Bot. 56: 87-94. http://dx.doi.org/10.1016/j.envexpbot.2005.01.008

del Amor FM, Ruiz-Sánchez MC, Martínez V and Cerdá A (2000). Gas exchange, water relations, and ion concentrations of salt-stressed tomato and melon plants. J. Plant Nutr. 23: 1315-1325. http://dx.doi.org/10.1080/01904160009382102

Garcia-Mas J, Benjak A, Sanseverino W, Bourgeois M, et al. (2012). The genome of melon (Cucumis melo L.). Proc. Natl. Acad. Sci. USA 109: 11872-11877.http://dx.doi.org/10.1073/pnas.1205415109

Gill SS and Tuteja N (2010). Reactive oxygen species and antioxidant machinery in abiotic stress tolerance in crop plants. Plant Physiol. Biochem. 48: 909-930. http://dx.doi.org/10.1016/i.plaphy.2010.08.016

Godfray HCJ, Beddington JR, Crute IR, Haddad L, et al. (2010). Food security: the challenge of feeding 9 billion people. Science 327: 812-818. http://dx.doi.org/10.1126/science.1185383

Hara K, Yagi M, Kusano T and Sano H (2000). Rapid systemic accumulation of transcripts encoding a tobacco WRKY transcription factor upon wounding. Mol. Gen. Genet. 263: 30-37. http://dx.doi.org/10.1007/PL00008673

Heinen RB, Ye Q and Chaumont F (2009). Role of aquaporins in leaf physiology. J. Exp. Bot. 60: 2971-2985. http://dx.doi. org/10.1093/jxb/erp171

Kawaura K, Mochida K and Ogihara Y (2008). Genome-wide analysis for identification of salt-responsive genes in common wheat. Funct. Integr. Genomics 8: 277-286. http://dx.doi.org/10.1007/s10142-008-0076-9

Khan MA, Ungar IA and Showalter AM (2000). Effects of salinity on growth, water relations and ion accumulation of the subtropical perennial halophyte, Atriplex griffithii var. stocksii. Ann. Bot. 85: 225-232. http://dx.doi.org/10.1006/ anbo.1999.1022

Laanemets K, Wang YF, Lindgren O, Wu J, et al. (2013). Mutations in the $S L A C l$ anion channel slow stomatal opening and severely reduce $\mathrm{K}^{+}$uptake channel activity via enhanced cytosolic $\left[\mathrm{Ca}^{2+}\right]$ and increased $\mathrm{Ca}^{2+}$ sensitivity of $\mathrm{K}^{+}$uptake channels. New Phytol. 197: 88-98. http://dx.doi.org/10.1111/nph.12008

Genetics and Molecular Research 15 (3): gmr.15038738 
Lin SH, Kuo HF, Canivenc G, Lin CS, et al. (2008). Mutation of the Arabidopsis NRT1.5 nitrate transporter causes defective root-to-shoot nitrate transport. Plant Cell 20: 2514-2528. http://dx.doi.org/10.1105/tpc.108.060244

Mangal J, Hooda P and Lal S (1988). Salt tolerance of five muskmelon cultivars. J. Agric. Sci. 110: 641-643. http://dx.doi. org $/ 10.1017 / \mathrm{S} 0021859600082241$

Mattana M, Biazzi E, Consonni R, Locatelli F, et al. (2005). Overexpression of Osmyb4 enhances compatible solute accumulation and increases stress tolerance of Arabidopsis thaliana. Physiol. Plant. 125: 212-223. http://dx.doi. org/10.1111/j.1399-3054.2005.00551.x

Munns R and Tester M (2008). Mechanisms of salinity tolerance. Annu. Rev. Plant Biol. 59: 651-681. http://dx.doi. org/10.1146/annurev.arplant.59.032607.092911

Qi Y, Liu W, Qiu L, Zhang S, et al. (2010). Overexpression of glutathione S-transferase gene increases salt tolerance of Arabidopsis. Russ. J. Plant Physiol. 57: 233-240. http://dx.doi.org/10.1134/S102144371002010X

Sandalio LM, Rodríguez-Serrano M, Romero-Puertas MC and del Río LA (2013). Role of peroxisomes as a source of reactive oxygen species (ROS) signaling molecules. Subcell. Biochem. 69: 231-255. http://dx.doi.org/10.1007/97894-007-6889-5 13

Sinetova MA, Mironov KS, Mustardy L, Shapiguzov A, et al. (2015). Aquaporin-deficient mutant of Synechocystis is sensitive to salt and high-light stress. J. Photochem. Photobiol. B 152 (Pt B): 377-382. http://dx.doi.org/10.1016/j. jphotobiol.2015.07.012

Singh K, Foley RC and Oñate-Sánchez L (2002). Transcription factors in plant defense and stress responses. Curr. Opin. Plant Biol. 5: 430-436. http://dx.doi.org/10.1016/S1369-5266(02)00289-3

Sirichandra C, Wasilewska A, Vlad F, Valon C, et al. (2009). The guard cell as a single-cell model towards understanding drought tolerance and abscisic acid action. J. Exp. Bot. 60: 1439-1463. http://dx.doi.org/10.1093/jxb/ern340

Sivritepe HÖ, Sivritepe N, Eriş A and Turhan E (2005). The effects of $\mathrm{NaCl}$ pre-treatments on salt tolerance of melons grown under long-term salinity. Sci. Hortic. 106: 568-581. http://dx.doi.org/10.1016/j.scienta.2005.05.011

Sun J, Chen SL, Dai SX, Wang RG, et al. (2009). Ion flux profiles and plant ion homeostasis control under salt stress. Plant Signal. Behav. 4: 261-264.http://dx.doi.org/10.4161/psb.4.4.7918

Sun L, Yu G, Han X, Xin S, et al. (2015). TsMIP6 enhances the tolerance of transgenic rice to salt stress and interacts with target proteins. J. Plant Biol. 58: 285-292. http://dx.doi.org/10.1007/s12374-015-0069-x

Tester M and Davenport R (2003). $\mathrm{Na}^{+}$tolerance and $\mathrm{Na}^{+}$transport in higher plants. Ann. Bot. 91: 503-527. http://dx.doi. org/10.1093/aob/mcg058

Villarino GH, Bombarely A, Giovannoni JJ, Scanlon MJ, et al. (2014). Transcriptomic analysis of Petunia hybrida in response to salt stress using high throughput RNA sequencing. PLoS One 9: e94651. http://dx.doi.org/10.1371/ journal.pone.0094651

Wang L, Wei S, Chen J, Zhang Y, et al. (2013). Regulation of the inward rectifying K $\mathrm{K}^{+}$channel MIRK and ion distribution in two melon cultivars (Cucumis melo L.) under NaCl salinity stress. Acta Physiol. Plant. 35: 2789-2800. http:// dx.doi.org/10.1007/s11738-013-1311-0

Wei S, Wang L, Zhang Y and Huang D (2013). Identification of early response genes to salt stress in roots of melon (Cucumis melo L.) seedlings. Mol. Biol. Rep. 40: 2915-2926. http://dx.doi.org/10.1007/s11033-012-2307-3

Wu H, Zhu M, Shabala L, Zhou M, et al. (2015). $\mathrm{K}^{+}$retention in leaf mesophyll, an overlooked component of salinity tolerance mechanism: a case study for barley. J. Integr. Plant Biol. 57: 171-185.http://dx.doi.org/10.1111/jipb.12238

Yong HY, Zou Z, Kok EP, Kwan BH, et al. (2014). Comparative transcriptome analysis of leaves and roots in response to sudden increase in salinity in Brassica napus by RNA-seq. BioMed Res. Int. 2014: 467395. http://dx.doi. org $/ 10.1155 / 2014 / 467395$

Yuan F, Lyu MJA, Leng BY, Zheng GY, et al. (2015). Comparative transcriptome analysis of developmental stages of the Limonium bicolor leaf generates insights into salt gland differentiation. Plant Cell Environ. 38: 1637-1657. http:// dx.doi.org/10.1111/pce.12514

Zhang YD, Véry AA, Wang LM, Deng YW, et al. (2011). A K ${ }^{+}$channel from salt-tolerant melon inhibited by $\mathrm{Na}^{+}$. New Phytol. 189: 856-868. http://dx.doi.org/10.1111/j.1469-8137.2010.03526.x

Zheng X, Chen B, Lu G and Han B (2009). Overexpression of a NAC transcription factor enhances rice drought and salt tolerance. Biochem. Biophys. Res. Commun. 379: 985-989. http://dx.doi.org/10.1016/j.bbrc.2008.12.163

Zhu Cy, Huang Df, Cai Bs, Yu Cx, et al. (2010). Salt tolerance and indicators assessment of melon germplasms in germination stage. J. Shanghai Jiaotong Univ. (Agr. Sci.) 6: 006.

\section{Supplementary material}

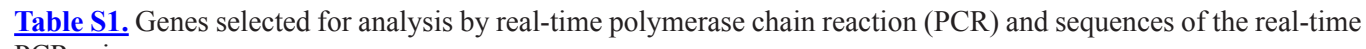
PCR primer.

Genetics and Molecular Research 15 (3): gmr.15038738 


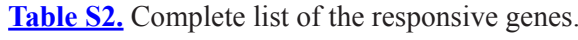

Table S3. Detailed Gene Ontogeny (GO) terms for differentially expressed genes (DEGs).

Table S4. Gene Ontogeny (GO) terms for differentially expressed genes (DEGs) in level 3.

Table S5. Annotated common up- and downregulated genes.

Table S6. Detailed Gene Ontogeny (GO) terms for common upregulated genes.

Table S7. Detailed Gene Ontogeny (GO) terms for common downregulated genes.

Table S8. Comparison of common upregulated and downregulated genes.

Table S9. Pathway analysis.

Table S10. Sequences of polymerase chain reaction (PCR) genes.

Table S11. Detailed annotation for BXC and YL.

Figure S1. Comparison of gene expression levels in BXC (A) and YL (B) with and without salt treatment. 